\title{
CONCERNING ADJUNCTIONS TO ALGEBRAS
}

BY J. L. DORROH*

In $\S 3$ of his paper, Algebras which do not possess a finite basis, $\dagger$ J. H. M. Wedderburn gives a set of postulates and definitions for an algebra. The question of the possibility of adjoining an identity to such an algebra arises. The purpose of the present paper is to show that this adjunction is always possible.

It may be seen from the arguments that Theorems 1 and 2 remain true if the term algebra be replaced by one implying a set of elements of which it is assumed only that it is an abelian group under addition and a semi-group under multiplication. The proof of Theorem 3 employs a distributive property, as will be indicated.

TheOREm $1 . \ddagger$ If $A$ is an algebra, then there exists an algebra $B$ which contains a proper, invariant subalgebra $A^{\prime}$ isomorphic with $A$ and an element $I$ not in $A^{\prime}$ such that, for every element $b$ of $B$, the relation $I b=b I=b$ holds.

If $x$ is an element of $A$ and $n$ is a positive rational integer, let $n x=x n$ denote the sum $x+x+\cdots+x$ ( $n$ summands) and let $(-n) x=x(-n)$ denote the same sum as $n(-x)$. Let $0 \cdot x$ $=x \cdot 0=0$.

Let $B$ denote the set of all pairs $(n, x)$ where $n$ is a rational integer and $x$ is an element of $A$. Suppose that $(n, x)+(m, y)$ $=(n+m, x+y)$. Then $B$ is an abelian group under addition. Let $(n, x)(m, y)=(n m, n y+m x+x y)$. Then $B$ is an algebra and the subset $A^{\prime}$ of $B$ consisting of all the elements of $B$ of the form $(0, x)$ is isomorphic with $A$ and is invariant in $B$. Let $I$ denote the element $(1,0)$ of $B ;(1,0)(n, x)=(n, x)(1,0)=(n, x)$ for every $n$ and $x$.

The multiplication in $B$ is distributive, associative and distributive, commutative, respectively, provided the same is true for $A$.

* National Research Fellow.

$\dagger$ Transactions of this Society, vol. 26 (1924), pp. 395-426.

$\ddagger$ For a proof of this theorem for an algebra with a finite basis, see, for example, L. E. Dickson, Algebras and their Arithmetics, 1923, p. 97.

$\S$ In this paper isomorphism means simple isomorphism. 
THEOREM 2. If an algebra $A$ has the properties

(1) if $x$ is an element of $A$ and $m$ is a positive rational integer, $A$ contains an element $y$ such that $m y=x$;

(2) if $x$ is an element of $A$ and $m$ is a positive rational integer and $x \neq 0$, we have $m x \neq 0$; then there exists an algebra $B$ which contains a proper, invariant subalgebra isomorphic with $A$ and a proper subalgebra isomorphic with the rational field.

If $x$ is an element of $A$ and $m$ is a positive integer, it follows from (1) that $A$ contains an element $y$ such $m y=x$; let $y$ be denoted by $x / m$; it follows from (2) that $x / m$ is unique.

If $r$ is a rational number, let $r=m / n$, where $m$ and $n$ are integers and $n$ is positive. Let $r x=x r=m(x / n)$ for each element $x$ of $A$. Then $r x$ is unique, for suppose that $m$ and $n$ have been chosen relatively prime and let $k$ denote a positive integer, then $k m(x /(k n))=m(x / n)$, for $k n[k m(x /(k n))]=k m x=k n[m(x / n)]$ and the equality of the brackets follows from (2).

Let $B$ denote the set of all pairs $(r, x)$ where $r$ is a rational number and $x$ is an element of $A$. Under the following rules for addition and multiplication $B$ is an algebra:

$$
\begin{aligned}
& (r, x)+\left(r^{\prime}, x^{\prime}\right)=\left(r+r^{\prime}, x+x^{\prime}\right), \\
& (r, x)\left(r^{\prime}, x^{\prime}\right)=\left(r r^{\prime}, r x^{\prime}+r^{\prime} x+x x^{\prime}\right) .
\end{aligned}
$$

The element $(1,0)$ of $B$ is its identity, since we have $(1,0)(r, x)$ $=(r, x)=(r, x)(1,0)$.

The set of all the elements of $B$ of the form $(0, x)$ is a proper, invariant subalgebra of $B$ and it is isomorphic with $A$.

The set of all the elements of $B$ of the form $(r, 0)$ is a proper subalgebra of $B$ isomorphic with the rational field.

The multiplication in $B$ is distributive, associative and distributive, commutative, respectively, provided the same is true for $A$.

THEOREM 3. If $A$ is an algebra subject to condition (2) of Theorem 2, there exists an algebra $B$ which contains a subalgebra isomorphic with $A$ and such that, if $b$ is an element of $B$ and $n$ is a positive rational integer, $B$ contains a unique element $c$ such that $n c=b$.

In the set $D$ of all pairs $(r, x)$, where $r$ is a rational number and $x$ is an element of $A,(r, x)$ will be said to be equivalent to $\left(r^{\prime}, x^{\prime}\right)$ if $s v x=t u x^{\prime}$, where $r=s / t$ and $r^{\prime}=u / v$, and where $s, t, u$ 
and $v$ are integers and $t$ and $v$ are positive. This relation will be denoted by $(r, x) \sim\left(r^{\prime}, x^{\prime}\right)$. From the hypothesis concerning $A$ it follows that the equivalence of $(r, x)$ and $\left(r^{\prime}, x^{\prime}\right)$ is independent of the choice of rational expressions for $r$ and $r^{\prime}$.

(1) $(r, x) \sim(r, x)$.

(2) If $(r, x) \sim\left(r^{\prime}, x^{\prime}\right)$, then $\left(r^{\prime}, x^{\prime}\right) \sim(r, x)$.

(3) If $(r, x) \sim\left(r^{\prime}, x^{\prime}\right)$ and $\left(r^{\prime}, x^{\prime}\right) \sim\left(r^{\prime \prime}, x^{\prime \prime}\right)$, then also $(r, x) \sim$ $\left(r^{\prime \prime}, x^{\prime \prime}\right)$. Let $r=s / t, r^{\prime}=u / v, r^{\prime \prime}=p / q ; s, t, u, v, p, q$ integers and $t>0, v>0, q>0$. If $s v x=t u x^{\prime}$ and $u q x^{\prime}=v p x^{\prime \prime}$, then 'svqx=tuqx' and $t u q x^{\prime}=t v p x^{\prime \prime}$; hence $s v q x=t v p x^{\prime \prime}$ and therefore $s q x=t p x^{\prime \prime}$.

Let $(r, x)+\left(r^{\prime}, x^{\prime}\right)=\left(1 /(t v), s v x+t u x^{\prime}\right)$, where $r=s / t, r^{\prime}=u / v$; $s, t, u, v$ integers and $t, v$ positive. If $\left(r^{\prime}, x^{\prime}\right) \sim\left(r^{\prime \prime}, x^{\prime \prime}\right)$, then $(r, x)+\left(r^{\prime}, x^{\prime}\right) \sim(r, x)+\left(r^{\prime \prime}, x^{\prime \prime}\right)$. Let $r^{\prime \prime}=p / q ; p, q$ integers and $q>0$. Then $(r, x)+\left(r^{\prime \prime}, x^{\prime \prime}\right)=\left(1 /(t q), s q x+p t x^{\prime \prime}\right)$. By hypothesis $u q x^{\prime}=v p x^{\prime \prime}$. Hence we have $t u q x^{\prime}=t v p x^{\prime \prime}$ and therefore $\left(1 /(t v q), \quad s v q x+t u q x^{\prime}\right) \sim\left(1 /(t v q), \quad s v q x+t v p x^{\prime \prime}\right), \quad$ but $(1 /(t v q)$, $\left.s v q x+t u q x^{\prime}\right) \sim\left(1 /(t v), \quad s v x+t u x^{\prime}\right)$ and $\left(1 /(t v q), \quad s v q x+t v p x^{\prime \prime}\right)$ $\sim\left(1 /(t q), s q x+t p x^{\prime \prime}\right)$. It follows that if $(r, x) \sim\left(r^{\prime \prime \prime}, x^{\prime \prime \prime}\right)$ and $\left(r^{\prime}, x^{\prime}\right) \sim\left(r^{\prime \prime}, x^{\prime \prime}\right)$, then $(r, x)+\left(r^{\prime}, x^{\prime}\right) \sim\left(r^{\prime \prime \prime}, x^{\prime \prime \prime}\right)+\left(r^{\prime \prime}, x^{\prime \prime}\right)$.

Let $(r, x)\left(r^{\prime}, x^{\prime}\right)=\left(r r^{\prime}, x x^{\prime}\right)$. Then if $\left(r^{\prime}, x^{\prime}\right) \sim\left(r^{\prime \prime}, x^{\prime \prime}\right)$, $(r, x)\left(r^{\prime}, x^{\prime}\right) \sim(r, x)\left(r^{\prime \prime}, x^{\prime \prime}\right)$. For by hypothesis $u q x^{\prime}=v p x^{\prime \prime}$ and hence $u q x x^{\prime}=v p x x^{\prime \prime}\left(r=s / t, r^{\prime}=u / v, r^{\prime \prime}=p / q\right.$ as before $)$, and

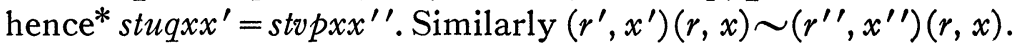
Hence if $(r, x) \sim\left(r^{\prime \prime \prime}, x^{\prime \prime \prime}\right)$, then $(r, x)\left(r^{\prime}, x^{\prime}\right) \sim\left(r^{\prime \prime \prime}, x^{\prime \prime \prime}\right)$ $\cdot\left(r^{\prime \prime}, x^{\prime \prime}\right)$.

For each rational number $w$ let $w(r, x)=(r, x) w=(w r, x)$.

For each $(r, x)$ of $D$ let $C_{(r, x)}$ denote the class of all pairs $\left(r^{\prime}, x^{\prime}\right)$ of $D$ such that $\left(r^{\prime}, x^{\prime}\right) \sim(r, x)$. Then $C_{(r, x)}=C_{\left(r^{\prime \prime}, x^{\prime \prime}\right)}$ if, and only if, $(r, x) \sim\left(r^{\prime \prime}, x^{\prime \prime}\right)$. Let $C_{(r, x)}+C_{\left(r^{\prime}, x^{\prime}\right)}=C_{(r, x)+\left(r^{\prime}, x^{\prime}\right)}$ and let $C_{(r, x)} C_{\left(r^{\prime}, x^{\prime}\right)}=C_{(r, x)\left(r^{\prime}, x^{\prime}\right)} \dagger$ These products and sums are unique. For each rational number $w$ let $w C_{(r, x)}=\left[C_{(r, x)}\right] w$ $=C_{w(r, x)}$.

* To establish this equation and the corresponding one implied by the succeeding expressions in the text, it is sufficient to assume that if $n$ is a positive rational integer and $x$ and $y$ are elements of $A$, then $x(n y)=n x y$ and $(n y) x$ $=n(y x)$. That this is a distributive property is seen from the definition of $n y$.

$\dagger$ For convenience the ordinary symbols for addition and multiplication are retained, although the operations are not the ones they would ordinarily indicate for classes. 
Let $B$ denote the collection of all the classes $C_{(r, x)}$. Then $B$ is an algebra. The subset $A^{\prime}$ of $B$ consisting of all the classes $C_{(1, x)}$ of $B$ is isomorphic with $A$ and is identical with $B$ if, and only if, for each element $x$ of $A$ and each positive rational integer $m, A$ contains an element $x / m$ such that $m(x / m)=x$.

If $C_{(r, x)}$ is an element of $B$ and $m$ is a positive rational integer, then $C_{(r / m, x)}$ is an element of $B$ and $m C_{(r / m, x)}=C_{(r, x)}$.

Multiplication in $B$ is associative, commutative, distributive, respectively, if the same is true for $A$.

Princeton University

\section{ON THE RANK OF THE PRODUCT OF CERTAIN SQUARE MATRICES*}

BY W. O. MENGE

1. Introduction. This paper presents several theorems which were found during an investigation conducted by the author into the structure of matrices which transform given matrices into their so-called classic and rational canonical forms. $\dagger$ When the elementary divisors of a given matrix are known, these theorems completely determine the rank of a product of matrices of the form

$$
\prod_{i=1}^{\omega}\left(A-\lambda_{i} I\right)^{k_{i}} .
$$

An interesting proof of the Hamilton-Cayley theorem and a determination of the equation of minimum degree satisfied by a matrix are obtained from this point of view.

2. Invariant Factors. Consider the square matrix $A=\left(a_{i j}\right)$ of order $n$ with constant elements. If the $n$-rowed identity matrix be denoted by $I$, the characteristic matrix $(A-\lambda I)$ is defined as the matrix obtained by subtracting the variable $\lambda$ from each principal diagonal element of $A$. The determinant, $D(\lambda)$, of the characteristic matrix $(A-\lambda I)$ is called the characteristic de-

* Presented to the Society, December 30,1930. The author wishes to acknowledge his appreciation to J. A. Nyswander, University of Michigan, for many helpful suggestions throughout the progress of the work.

$\dagger$ Dickson, Modern Algebraic Theory, Chap. 5. 